\title{
Coarse-Grained Modeling of Protein Dynamics
}

\author{
Sebastian Kmiecik ${ }^{*}$, Jacek Wabik, Michal Kolinski, \\ Maksim Kouza, and Andrzej Kolinski
}

\begin{abstract}
Simulations of protein dynamics may work on different levels of molecular detail. The levels of simplification (coarse-graining) can range from very low to atomic resolution and may concern different simulation aspects (including protein representation, interaction schemes or models of molecular motion). So-called coarse-grained (CG) models offer many advantages, unreachable by classical simulation tools, as demonstrated in numerous studies of protein dynamics. Followed by a brief introduction, we present example applications of CG models for efficient predictions of biophysical mechanisms. We discuss the following topics: mechanisms of chaperonin action, mechanical properties of proteins, membrane proteins, protein-protein interactions and intrinsically unfolded proteins. Presently, these areas represent emerging application fields of CG simulation models.
\end{abstract}

Abbreviations: CG, coarse-grained; MD, Molecular Dynamics; MC, Monte Carlo.

\section{$1 \quad$ Introduction}

The steady increase in computational power constantly sets new limits in simulations of biomolecular dynamics (Vendruscolo and Dobson 2011). Nevertheless, the majority of biologically relevant protein dynamic processes remain beyond the reach of atomistic Molecular Dynamics (MD), the classical simulation tool

Sebastian Kmiecik · Jacek Wabik · Maksim Kouza · Andrzej Kolinski

Laboratory of Theory of Biopolymers, Faculty of Chemistry, University of Warsaw,

Warsaw, Poland

e-mail: sekmi@chem.uw.edu.pl

Michal Kolinski

Bioinformatics Laboratory, Mossakowski Medical Research Centre Polish

Academy of Sciences, Warsaw, Poland

* Corresponding author.

A. Liwo (Ed.): Comput. Methods to Study the Struct. \& Dyn. of Biomolecules, SSBN 1, pp. 55-79. DOI: 10.1007/978-3-642-28554-7_3

(C) Springer-Verlag Berlin Heidelberg 2014 
(Kmiecik et al. 2011). In such cases, the introduction of properly designed simplifications that capture relevant physical features can be the only option, or incomparably cheaper than atomistic MD, to better understand macromolecular processes.

A variety of purely theoretical models for analyzing the dynamic properties of proteins have been proposed (Munoz et al. 1998; Wolynes et al. 1995). Nevertheless they appeared to be rather limited in their predictions. This is due to the complicated nature of proteins and rules governing their structure. Compared to purely analytical methods, the molecular simulation approach is better suited to handling protein complexity. Presently, molecular simulations represent a powerful and the most widely used theoretical approach for the understanding of protein dynamics.

\subsection{CG Simulation Models}

The most direct computational approach to protein dynamics prediction is simulation of a dynamic system of interest. A simplified simulation model of proteins is probably the earliest example of CG approach in structural biology, developed in the mid-1970s (Levitt and Warshel 1975). Since that time the field has grown tremendously, branching out in many variants of protein representation, interaction potentials and sampling models (Figure 1). Interestingly, recent estimates indicate a significant increase in the number of studies that rely on CG simulations (based on publication statistics over the last decade) (Takada 2012). This significant rise is perhaps related to a growing number of experimentally solved structures of large biomolecules (or their complexes), too large to be reasonably addressed by all-atom simulations.

A number of mean-resolution CG models have been developed for protein structure prediction (Kolinski and Skolnick 2004). Some of them enable efficient simulation of dynamic processes. A typical example is the CABS model (Kolinski 2004), which acronym stands for the united atoms representing a single residue in a protein chain (CA -alpha carbon of the main chain, cB -beta carbon, and $\mathrm{S}$-the center of side group). Thus, in the CABS model, a single amino acid is represented by 2-4 (depending on the side-chain size) interaction centers, and one of them (C-alpha) is placed into a high-resolution lattice. The interaction scheme is based on mean-force potentials derived by the statistical analysis of known protein structures. In spite of the fact that the interaction scheme is obtained only from known crystallographic structures related to completely random protein chains, protein dynamics processes (folding, unfolding, diffusion, etc.) simulated by the CABS method are qualitatively correct (Kmiecik et al. 2007; Kmiecik and Kolinski 2008, 2011; Kmiecik et al. 2012). These qualitatively correct results, not trivial to obtain, show that interaction patterns of unfolded (or partially unfolded) proteins are quite similar to the interactions seen in fully folded structures. The lattice representation of CABS proteins significantly increases the speed of conformational updates. Simulation processes are controlled by the Monte Carlo (MC) scheme: random series of local conformational transitions. This pseudorandom Monte Carlo process does not describe accurately ultra-short-time motions and ranges of a few angstroms, although longer-time (and space) dynamics is 


\section{A. Protein representation}

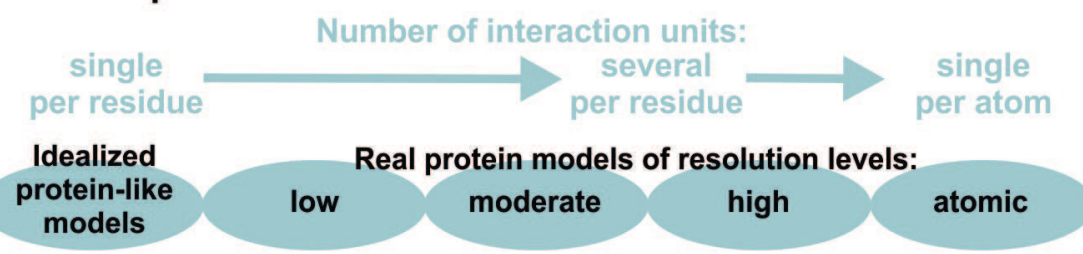

\section{B. Interaction potential}

Complexity levels:

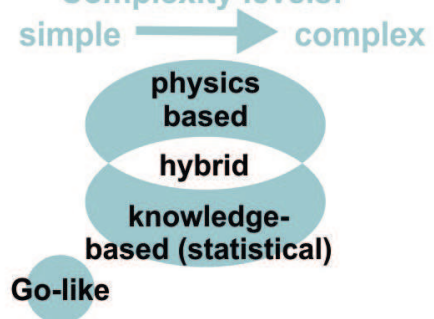

\section{Sampling model}

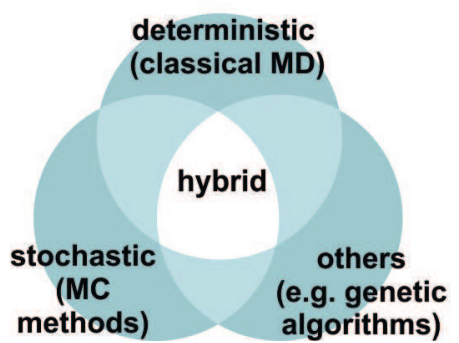

Fig. 1 Conceptual components of CG protein simulation models and their variants: (A) protein representation, (B) interaction schemes (Go-like potentials are protein specific, i.e., native interactions are favored to assure the lowest energy for the native conformation, and are used individually or in combination with non-protein specific: physics- or knowledgebased schemes), (C) sampling models. This diagram applies either to continuous-space or discrete (lattice) models. For detailed review of these variants and coarse-graining levels refer to (Kolinski and Skolnick 2004).

essentially identical with continuous space models (Kolinski 2004). The coarsegraining of $\mathrm{CABS}$ enables very fast derivation of its low resolution from high resolution atomistic coordinates, and what is more important quite accurate allatom structures could be very rapidly re-computed from CABS coordinates (Kmiecik et al. 2007; Kmiecik et al. 2012).

\subsection{From CG to All-Atom Structures: Multiscale Modeling}

One of the major future issues of CG dynamics studies is the design of methods for the reliable and efficient transition between simplified and atomic resolution levels (Scheraga et al. 2007), as the element of multiscale methodologies. The idea of multiscale modeling is efficient computation on a CG scale to send it to the detailed all-atom simulation, or vice versa (Kmiecik et al. 2011). Obviously, the CG model used in the multiscale methods must produce physically realistic coarse-grain protein structures. Even if it is fulfilled, it is a non-trivial problem to add all-atom details to CG structures to produce physically realistic all-atom counterparts (Kmiecik et al. 2007). It has been demonstrated in applications to protein folding CG trajectories that reliable and efficient movement between CG and atomic resolution is feasible (Kmiecik et al. 2012; Heath et al. 2007). Finally, it is accepted that one of the most promising future directions is to develop approaches 
that can minimize the difference between the simplified and atomic models (Kamerlin et al. 2011).

\section{Applications in Structural Biology}

In this section, we discuss several recent examples of CG modeling, including our reports and other published literature. The section covers the following actively studied tasks of protein dynamics: mechanisms of chaperonin action, mechanical properties of proteins, dynamics of protein-protein interactions and membrane and intrinsically unfolded proteins. We consider these aspects of protein dynamics one of the most prospective development areas of new computational strategies including CG protein simulation models. Most of our own examples of CG simulations described below were done using the CABS CG modeling tool (Kolinski 2004) (the CABS applications to the study of protein folding pathways have been reviewed elsewhere (Kmiecik et al. 2011)).

\subsection{Testing Mechanisms of Macromolecular Dynamics via Simple Models: Chaperonin Action}

Complex macromolecular processes can be generalized to very simple concepts and tested computationally on a very general level. This is the case of the studies of chaperonin action. Chaperonin and its protein substrate is a very large protein complex whose dynamic processes are way beyond the reach of classical dynamics simulation models. Over the past 20 years a significant number of studies, both experimental and theoretical, have been pursued to understand how chaperonins (like GroEL) facilitate protein folding processes in the cell.

Many theoretical models have been proposed focusing either on the passive (aggregation prevention) or active (folding promotion) possible roles of chaperonins (Jewett and Shea 2009). A number of CG simulation studies investigated the effect of confinement on protein folding using very simplistic (Zhou and Dill 2001), simple lattice (Betancourt and Thirumalai 1999), off lattice C-alpha based and Go-like (Takagi et al. 2003; Rathore et al. 2006) or more realistic (Baumketner et al. 2003) models. Another aspect of chaperonin action, namely the effect of interactions of the protein substrate with chaperonin cavity, was also a subject of numerous CG simulations studies, e.g., by lattice (Betancourt and Thirumalai 1999) and off-lattice (Jewett et al. 2004) models. For broad, recent reviews covering the use of CG models in chaperonin action studies, see (Jewett and Shea 2009; Lucent et al. 2009).

Probably the most popular theoretical model which provides explanation of the chaperonin active role is the Iterative Annealing Model (IAM). In this model chaperonin promotes folding by unfolding the protein substrate from misfolding traps through its hydrophobic interactions with cage walls. Just very recently, we have attempted to test the IAM hypothesis using a de novo CABS modeling approach employing a non-specific (non-Go-like) knowledge-based interaction scheme (Kmiecik and Kolinski 2011). Importantly, in most (if not all, as described by 
Lucent et al. (Lucent et al. 2009)) simulation studies testing various chaperonin models on real (i.e. not too much simplified) protein substrates, a common simplified interaction model was used: the Go-like model. Therefore, in contrast to earlier simulation studies, the CABS model did not preclude transient conformers stabilized by non-native interactions.

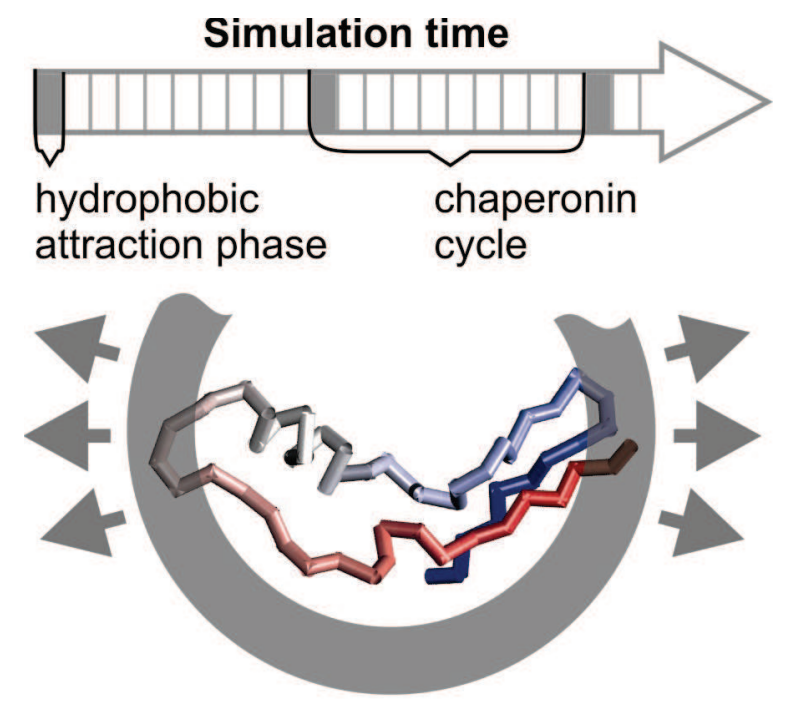

Fig. 2 A simple chaperonin model used in protein folding studies with the CG CABS model (Kmiecik and Kolinski 2011). The chaperonin cage was simulated as a sphere with a thick wall of variable hydrophobicity. In the basic state the walls are inert for 9/10 of the simulation time. Periodically (see the simulation timescale above in the Figure) the walls became hydrophobic, attracting the encapsulated protein chain with a strength typical for hydrophobic interactions within folded proteins (according to the CABS force field).

The CABS simulation results showed that periodic distortion of the simulated proteins by hydrophobic chaperonin interactions promotes rapid folding and leads to a decrease in folding temperature. According to the observed mechanism of folding promotion, chaperonin prevents kinetically trapped conformations. This is contrary to the so far accepted interpretation of the IAM model suggesting not the prevention but rather the unfolding action from already trapped conformations. Interestingly, the analysis of the folding trajectories enables general observation of chaperonin-induced modulation of the observed folding mechanisms from nucleation-condensation to more framework-like. All these observations remain in agreement with the experimental data on chaperonin-bound protein substrates, generally indicating an ensemble of compact and locally expanded states lacking stable tertiary interactions.

It is worth to mention that theoretical studies of chaperonin-mediated folding may have important conceptual applications in other fields (Lucent et al. 2009), e.g., in the development of structure-refinement software or in the construction of chaperonin-like molecules designed for desired biotech and medical applications. 
We have to realize that we are only at the beginning of the understanding of how chaperonins work. As pointed out by Lucent at al. (Lucent et al. 2009), so far most theoretical and experimental research focused on GroEL, a specific prokaryotic chaperonin. Since chaperonins exhibit different modes of action in prokaryotic and eukaryotic organisms, the investigation of these differences may be essential for the complete understanding of underlying mechanisms and protein folding itself. This challenging issue has already been addressed by a very simple lattice model (Jacob et al. 2007).

\subsection{Mechanical Unfolding of Proteins}

One of the functional features of proteins is response to a wide range of applied forces. Being subjected to an applied load, proteins play key roles in cytoskeletal organization (Fletcher and Mullins 2010), mechanics (Granzier and Labeit 2004), cellular transport (Serohijos et al. 2006), signaling (Stossel et al. 2001) and protein degradation (Hanson and Whiteheart 2005). The required external force to unfold protein is in the order of pico-Newtons. Since the atomic force microscopy (AFM) and laser optical tweezers (LOT) techniques (Rief et al. 1997; Marszalek et al. 1999; Simmons et al. 1996) detect forces in the pico-Newton range, they are useful tools for studying mechanical unfolding of biomolecules. In both techniques two major strategies are used. In the first technique, protein is pulled by a force ramped linearly with time, while monitoring the force (mechanical resistance) as a function of the end-to-end distance. The second strategy is the application of a constant force through force clamp devices. In experiments at a constant pulling speed, the total force experienced by protein is $\mathrm{F}=\mathrm{k}(\mathrm{vt}-\mathrm{x})$, where $\mathrm{k}, \mathrm{v}, \mathrm{t}$ and $\mathrm{x}$ are respectively: the spring constant (stiffness) of cantilever, pulling speed, time, and displacement of the pulled amino acid from its original position. Typically, in AFM experiments $\mathrm{k}$ and $\mathrm{v}$ are in the range of $10-1000 \mathrm{pN}$ and $10^{-11}-10^{-7} \AA / \mathrm{ps}$, respectively. In LOT, the velocity range is similar to that of AFM, whereas the typical values of spring constant, $\mathrm{k}=0.001-0.1 \mathrm{pN} / \mathrm{nm}$. Stiffness defines the force resolution of experiment. Thus, AFM can probe unfolding of strong proteins with required Fmax of about few hundreds of pN (such as titin (Rief et al. 1997) or ubiquitin (Carrion-Vazquez et al. 2003)), while LOT is precise enough for studying biomolecules with few tens of $\mathrm{pN}$ mechanical resistance (weaker proteins as well as DNA and RNA molecules (Smith et al. 1996; Liphardt et al. 2001)).

Figure 3A shows the force-extension profile obtained by constant velocity stretching experiments for Ig8 titin fragment. The peaks in Figure 3A are associated with breaking hydrogen bonds (HBs) between strands A' and G (Figure 3B) in single titin domains of the multidomain construction. Apart from molecular interactions studies, AFM technique can also be used to investigate the mechanical stability of proteins measured by $\mathrm{F}_{\max }$ in the force-extension profile (note that $\mathrm{F}_{\max }$ depends on the pulling speed logarithmically, $F_{\max } \sim \ln (\mathrm{v})$ (Evans and Ritchie 1997)). Measuring the mechanical stability in different solutions, one can also 


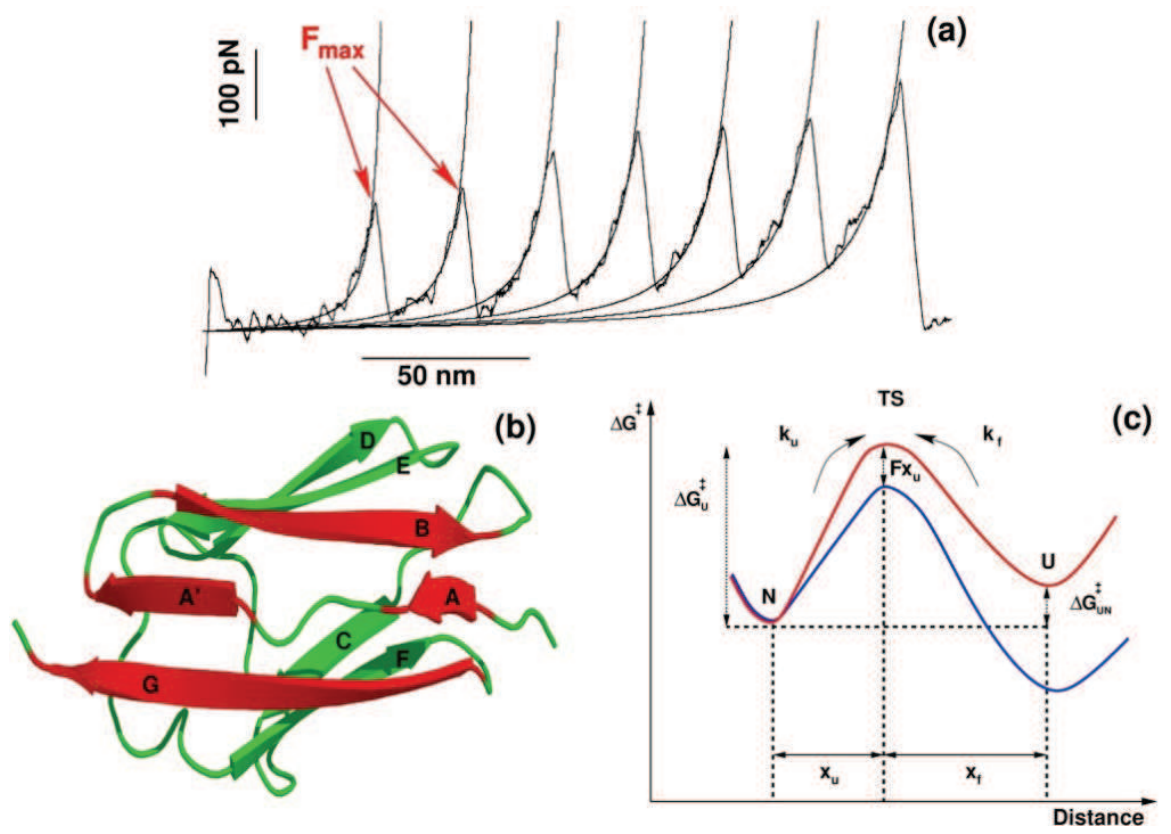

Fig. 3 (a) Force-extension profile obtained by stretching of Ig8 titin fragment (adapted from Ref. (Rief et al. 1997)). Each peak corresponds to unfolding of a single domain with maximum resisting force to stretching, $\mathrm{F}_{\max }$. Smooth curves are fits to the wormlike chain model. (b) Cartoon representation of native state conformation of I27 domain (PDB code: 1tit) with eight $\beta$-strands labeled: A (4-8), A'(11-15), B(18-25), C(32-36), D(47-52), E(55-61), $\mathrm{F}(69-75), \mathrm{G}(78-88)$. Importance of HBs between beta-strands marked by red color is described in the text. (c) Conceptual plot for the free energy landscape of protein unfolding without (red) and under (blue) the external force. An applied force lowers unfolding barrier by $\mathrm{Fx}_{\mathrm{u}}$ increasing exponentially the unfolding rate constant $\left(\mathrm{k}_{\mathrm{u}}\right)$, but decreasing exponentially the folding rate constant $\left(\mathrm{k}_{\mathrm{f}}\right) . \mathrm{x}_{\mathrm{u}}$ is the distance between native and transition state and $\mathrm{x}_{\mathrm{f}}$ is the distance between transition and denatured state.

probe the effect of environment on hydrogen bonding ( $\mathrm{Lu}$ and Schulten 2000). Moreover, the mechanical unfolding studies enable insights into: forces that drive biological processes, ligand binding affinity to proteins/receptors (Florin et al. 1994), force-induced intermediate states (Fowler et al. 2002; Li et al. 2005; Schwaiger et al. 2004), the mechanical unfolding free energy landscape (FEL) of proteins (Bustamante et al. 2004). The problem of FEL is considered in more detail below.

A major limitation of AFM experiments is that it cannot give the detailed characterization of conformational changes under the applied force at the atomic level. Computer simulations may be employed as a tool to complement experimental studies. Schulten's group used the all-atom models with an explicit water to study the mechanical unfolding of the I27 protein (Lu et al. 1998). They deciphered in great detail the unfolding pathway of I27 and demonstrated the existence of hump 
due to breaking HBs between beta strands A and B (Figure 3B) (Lu and Schulten 2000). Mechanical unfolding of a number of proteins has been also probed by allatom simulations with implicit solvent (Paci and Karplus 2000). The major shortcoming of all-atom MD simulations is that the pulling speed is about 6 orders of magnitude higher than that used in AFM experiments. It is unclear if in silico results obtained in such extreme conditions are meaningful to understand experiments (strong forces may considerably disturb FEL), although recent studies claimed that unfolding pathways are not sensitive to pulling forces and speeds (Lichter et al. 2012; Lee et al. 2009).

The timescale discrepancy (and the related discrepancy in stretching forces required to induce unfolding) between AFM experiments and simulation can be reduced by the usage of CG models. Nowadays GPU technique allows one to reach experimental pulling speeds by CG Go models (Zhmurov et al. 2010). CG Go models have been successfully used by many groups to study mechanical properties of proteins (West et al. 2006; Cieplak et al. 2002; Arad-Haase et al. 2010; Best and Hummer 2008). Despite their simplicity, in many cases they correctly capture unfolding pathways, FEL and mechanical stability of proteins. For example, a complete description of mechanical unfolding pathways of single and multidomain Ubiquitin at the level of secondary structure was obtained ( $\mathrm{Li}$ et al. 2007). It was shown that thermal and mechanical pathways for fibronectin type III and I27 domain are different (Paci and Karplus 2000). This is because the thermal fluctuations have more global effect on entire protein and unfold the most unstable part of protein while the force should propagate protein unwinding from the points to which force is applied. Having used Go-model, mechanical unfolding pathways of protein DDFLN4 (Li and Kouza 2009) and two slipknotted proteins (pdb codes - 1e2i and 1p6x) (Sulkowska et al. 2009), were shown to depend on the pulling speed.

The CG Go-models may be suitable for deciphering the FEL (Figure 3C). Considering FEL as a function of end-to-end distance, one can use Bell-approximation (Bell 1978) to estimate the distance between the native state (NS) and transition state (TS), $\mathrm{x}_{\mathrm{u}}$, using either the dependences of unfolding rates on the external force (Bell 1978) or the dependence of force on pulling speed (Evans and Ritchie 1997). The distance between the NS and TS $\mathrm{x}_{\mathrm{u}}$ (Figure 3C), estimated by the C-alpha Golike model (Clementi et al. 2000), was in excellent agreement with experimental results (Kouza et al. 2008; Caraglio et al. 2010). Furthermore, Li showed that $x_{u}$ (Figure 3C) is defined by the secondary structure content and approximately depends linearly on the contact order (Li 2007; Kumar and Li 2010), thus the helix proteins have larger distances from the native state to the transition state than beta proteins. It should be noted that the phenomenological Bell theory is based on the assumption that $\mathrm{x}_{\mathrm{u}}$ is not moving under stretching. Recently, applying Kramers theory (Kramers 1940) and assuming that the distance between NS and TS is force-dependent, Dudko et al. (Dudko et al. 2006) have gone beyond the Bell assumption. With the help of proposed nonlinear kinetic theory (Dudko et al. 2006) one can estimate not only intrinsic rate coefficient, $\mathrm{k}_{\mathrm{u}}$, and the distance between NS and TS, $\mathrm{x}_{\mathrm{u}}$, but also the unfolding barrier, $\Delta G_{\mathrm{u}}{ }^{++}$(Figure $3 \mathrm{C}$ ). 
One of the most successful application area of CG Go models were estimations of the mechanical stability of proteins (Sulkowska and Cieplak 2007; Sikora et al. 2011; Li 2007; Brockwell et al. 2003). It has been found that helix proteins are less stable than beta proteins and unfolding force $F_{\max }$ may be expressed as a linear function of the contact order (Plaxco et al. 1998). This is understandable because beta proteins have more long-range contacts leading to higher resistance to external perturbation (Kumar and Li 2010). Having used the Go models, Cieplak et al. have computed $\mathrm{F}_{\max }$ for thousands of proteins (Sikora et al. 2011; Sulkowska and Cieplak 2007) and found that the mechanical clamp (resistance-determining region of a protein) of the top strongest proteins is not only consisted of hydrogen bonded $\beta$-strands being sheared during the pulling. Structures tied by disulfide bonds were found to contribute to significantly larger mechanical stability than shear-based mechanical clamps. Novel mechanical clamps were identified and classified (Sikora et al. 2011; Sikora and Cieplak 2011). Later on, the high resistance to stretching of top 13 proteins (cysteine-slipknots) was confirmed by allatom steered molecular dynamics (SMD) simulations (Peplowski et al. 2011) and observed experimentally (Valbuena et al. 2009). Recently CG model was successfully applied even for proteins with non-trivial structures (Sulkowska et al. 2009; Sulkowska et al. 2010), which was confirmed by experiment (He et al. 2012). For a more detailed review of protein mechanostability, see Chapter 10 of this book entitled "Mechanostability of proteins and virus capsids."

The success of CG Go models is possibly associated with the fact that the pulling starts from the native state while these models are based on topology of the native state. However, in particular cases one has to be careful with predictions followed from these simple models. In the case of DDFLN4 protein, the Go model did not give the peak in the force-extension curve observed in the experiment. It was shown that the occurrence of that peak is due to non-native interactions neglected in Go model (Kouza et al. 2009). Thus, in certain cases the non-native interactions are important because non-native contacts appear during the unfolding process leading to intermediate states. To avoid possible artifacts associated with neglecting non-native interactions, CG models with more realistic potentials may be used. A good example of such case is the force-induced intermediate of Ubiquitin, which was neglected in Go-model simulations (Li et al. 2007), but detected by the Lund force-field (Irback et al. 2005).

SMD simulations have been used for a wide variety of applications in studies of biological processes and various biomolecules (Lee et al. 2009). Going forward, there is a clear need to move SMD techniques into living cell where proteins are exposed to their native (crowded) environment (Vogel and Sheetz 2006). One of the recent applications of SMD is to understand the mechanism of virus binding to its host cell (Sieben et al. 2012). Another issue of great interest is the application of SMD for studying the response of protein to periodic forces (Szymczak and Janovjak 2009). It is also worth to mention some important problems for further studies. For instance, it remains unclear if the distance between the native and transition states (distance $\mathrm{x}_{\mathrm{u}}$, see Figure 3C) followed from the nonlinear 
theory(Dudko et al. 2006) depends linearly on contact order (as it was obtained in the linear Bell approximation). Generally, the deciphering FEL is done by its projection onto one-dimensional space, usually end-to-end distance. However, the validity of such approximate mapping is not always true (Best et al. 2008), thus this issue requires further investigation.

\subsection{Dynamics of Protein-Protein Interactions}

Dynamics of protein-protein interactions is in the spotlight of current biomedical research. Molecular level descriptions of interaction dynamics give insights into relevant biological processes, underlying causes of some diseases or drug-receptor interactions.

Although CG models are less accurate than all-atom approaches, when used, we can focus on the important features of the thermodynamics and kinetics of protein complexes (Kim and Hummer 2008; Kim et al. 2008; Okazaki et al. 2012; Saunders and Voth 2012; Frembgen-Kesner and Elcock 2010). For example, Kim and Hummer (Kim and Hummer 2008) investigated binding affinities of Vps27 complexes with ubiquitin attached to the membrane, where folded domains were rigid and linkers between them were flexible. They used a C-alpha model with various variants of potentials for interactions between domains, linker movement and the protein-membrane complex. Predicted binding affinities, for various modeled complexes, were in good agreement with the experimental data. Furthermore, conformations of some ubiquitin complexes were predicted with very good preci-

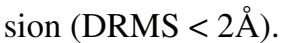

Interestingly, accurate values of binding affinities could also be determined with a more simplified model (Frembgen-Kesner and Elcock 2010). In this case the Brownian dynamics of the Barnase-Barstar complex was derived with a model in which three amino acids were represented by one bead. Computed kinetic data of the association process corresponded well with the experiment.

The binding of a protein coactivator to an enzyme is a class of extremely important processes, usually difficult to study due to relatively high protein rearrangements. The characterization of such processes has recently been the aim of CG simulation studies (Di Fenza et al. 2009; Kurcinski and Kolinski 2010). In one of them, Kurcinski and Kolinski (Kurcinski and Kolinski 2010) applied the CABS model to describe the activation of the Retinoid X Receptor (RXR) by 9-cis retinoic acid and the TRAP220 coactivator. They focused on specific transition states. The results agreed well with the experimental data and a two-stage sequential reaction mechanism could be suggested. Interestingly, the simulations were conducted with a fully flexible peptide coactivator (11 residues) and a moderately flexible receptor (238 residues) whose conformation was restrained to its experimental structure (see Figure 4 for the scheme of the multistage procedure). The resulting extent of conformational sampling was incomparably larger than with any classic all-atom simulation. 


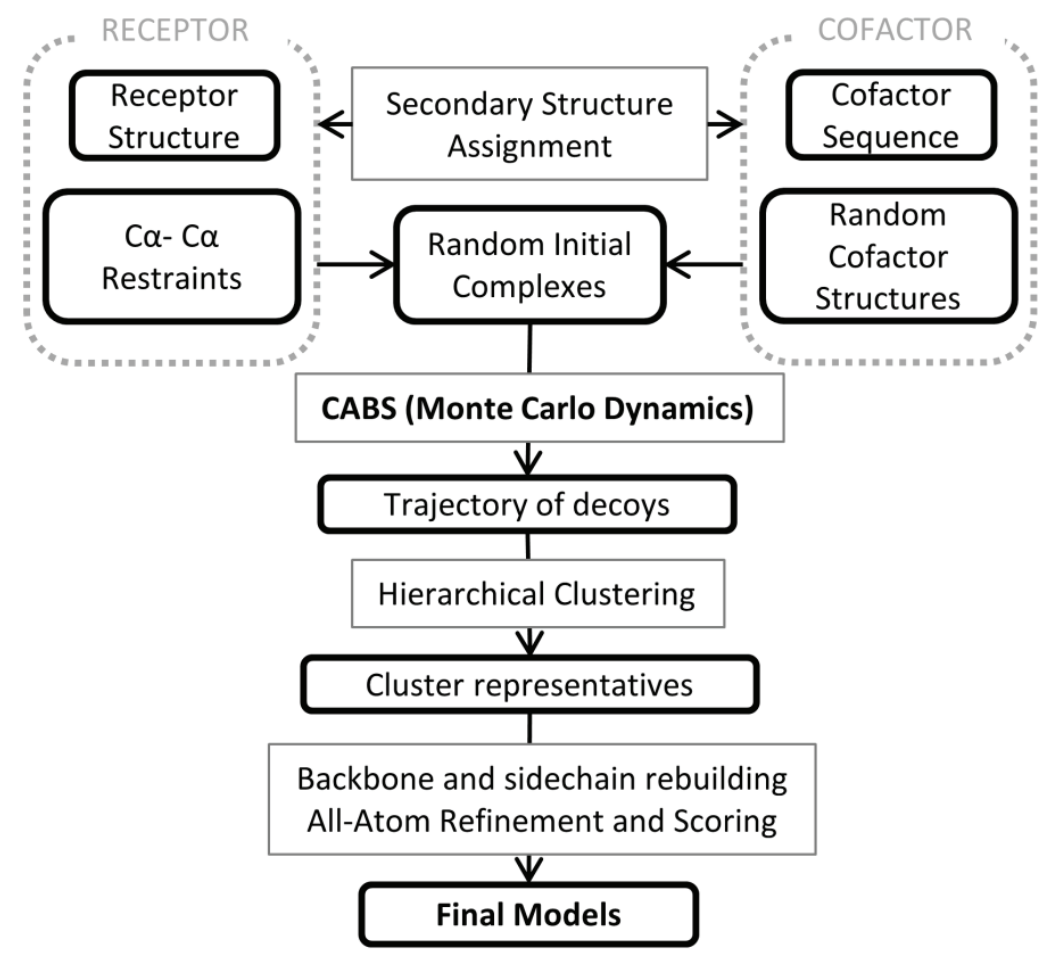

Fig. 4 Multiscale procedure for the description of binding between the Retinoid X Receptor (RXR) and the peptide (TRAP220) cofactor using CABS CG dynamics (Kurcinski and Kolinski 2010). The procedure starts from the generation of input data for a receptor and a protein cofactor. In the next step, the receptor and the cofactor are put together in many random configurations, subsequently subjected to CABS CG simulation. Various types of data along the procedure are shown in bold frames, while the applied computational methods in thin frames.

Apart from the possibility to use restraints from experimental structures to maintain the protein fold we can also use an elastic network model (ENM) as was done in Hall and Sansom (Hall and Sansom 2009). In this study proper structures of the Cohesin (162 residues)-Dockerin (60 residues) complex were predicted with a CG-Molecular Dynamics (CG-MD) model in which each amino acid was represented by four beads. $\mathrm{Ca}$. $80 \%$ of interfacial residues were identified correctly and two various ways of ligand binding were identified which agreed well with the results of experimental data.

With regard to the large-scale dynamics of protein systems, another promising and presently active field is CG dynamics of actin filaments (Chu and Voth 2006; Bindschadler 2010). Because of the scale of the system, it is extremely challenging to simulate myosin binding to actin filaments by all-atom MD. A multiscale model (Taylor and Katsimitsoulia 2010) enabled the observation of the myosin motor and an insight into its action. In this case, three levels of coarse-graining 
were introduced: chains of secondary structure elements, domains and molecules. The movement of each component was simulated by Brownian Dynamics. A more detailed, physicochemical view of the myosin-actin complex was recently obtained with a CG simulation model (Okazaki et al. 2012) in which each bead represented a single amino acid. In this case conclusions regarded also more general thermodynamic aspects of protein-protein association.

Another popular and important protein-protein dynamics issue, in which diverse levels of coarse-graining are applied, is protein aggregation. In the simplest models, a single unit (cuboid (Zhang and Muthukumar 2009) or tube (Auer et al. 2008)) represents the whole peptide, while in the most detailed models each amino acid consists of a few pseudo-atoms. For the recent review of these approaches, see (Wu and Shea 2011). Dramatic progress has been recently achieved in the CG modeling of large polyprotein complexes (made up of many copies of the same or different proteins) (Saunders and Voth 2012). In their review, Saunders and Voth present two general classes of CG methods: mapping methods that transfer information from one level to another only during parameterization and bridging methods that connect different scales of representation during simulation.

The major challenge in modeling of protein interaction dynamics seems to be as that recently outlined in the review of the performance of protein docking techniques (Vajda and Kozakov 2009). Namely, it is the treatment of substantial conformational changes. CG simulation models offer perhaps the most prospective means for modeling of extensive backbone dynamics in the nearest future.

\subsection{Dynamics of Membrane Proteins}

Membrane proteins play an important role in cell biology. They are responsible for signaling, molecular transport across lipid bilayers, maintaining cell structural stability and control of cell-cell interactions. Although $20 \%$ to $30 \%$ of all ORFs are predicted to encode membrane proteins, less than $1 \%$ of all known 3D protein structures account for membrane proteins (Nilsson et al. 2005). Moreover, those proteins are embedded in different types of lipid bilayers. The interaction with lipids is essential for both protein function (e.g. can affect integral membrane protein activity (Lee 2004)) and membrane properties such as hydrophobic thickness or lipid composition (Hunte 2005)). The complex nature of membrane-protein systems makes CG Molecular Dynamics (CG-MD) simulations a valuable approach to the investigation of dynamics, structure-function relationship and stability of membrane-protein systems. One of the best performing, and probably the most recognized, CG-MD approaches is based on the MARTINI force field (Marrink et al. 2007) that uses four-to-one atom mapping. Only four main types of interaction sites are defined: polar $(\mathrm{P})$, non-polar $(\mathrm{N})$, apolar $(\mathrm{C})$ and charged $(\mathrm{Q})$. Each particle type has a number of subtypes allowing accurate representation of solvent, protein and membrane structures. This approach enables treatment of very large systems (corresponding to systems consisting of more than 500,000 atoms) and offers timescales above $100 \mu$ s which are far beyond the scope of classical allatom-MD. The method was successfully applied by Sansom and co-workers for the prediction of protein positions within lipid bilayers (Scott et al. 2008). 
Self-assembly CG-MD simulations, starting from a protein surrounded by randomly positioned water and lipid molecules, were conducted for 91 different protein systems. The resulting structures gave insights into direct protein-lipid interactions, membrane distortion around different proteins and localization of proteins in the lipid bilayers, in agreement with experimental data (see Figure 5).

CG-MD simulations applying the MARTINI force field were also used for the investigation of helix associations and dimerization of membrane proteins. Sengupta and coworkers conducted a set of CG-MD simulations, each lasting $25 \mu \mathrm{s}$, to study the association mechanism of glycophorin A and two disruptive mutants, T87F and a triple mutant of the GxxxG motif (G79LG83LG86L), embedded in a DPPC lipid membrane model (Sengupta and Marrink 2010). In each case, dimers formed within the first $5 \mu \mathrm{s}$. The wild-type dimer packed in a right-handed manner, and the structure was consistent with the native structures defined by NMR studies (Smith et al. 2002). The analysis of free energy profiles reveals that two dimers formed by mutated peptides were less stable, by about $8-10 \mathrm{~kJ} \mathrm{~mol}^{-1}$ as a

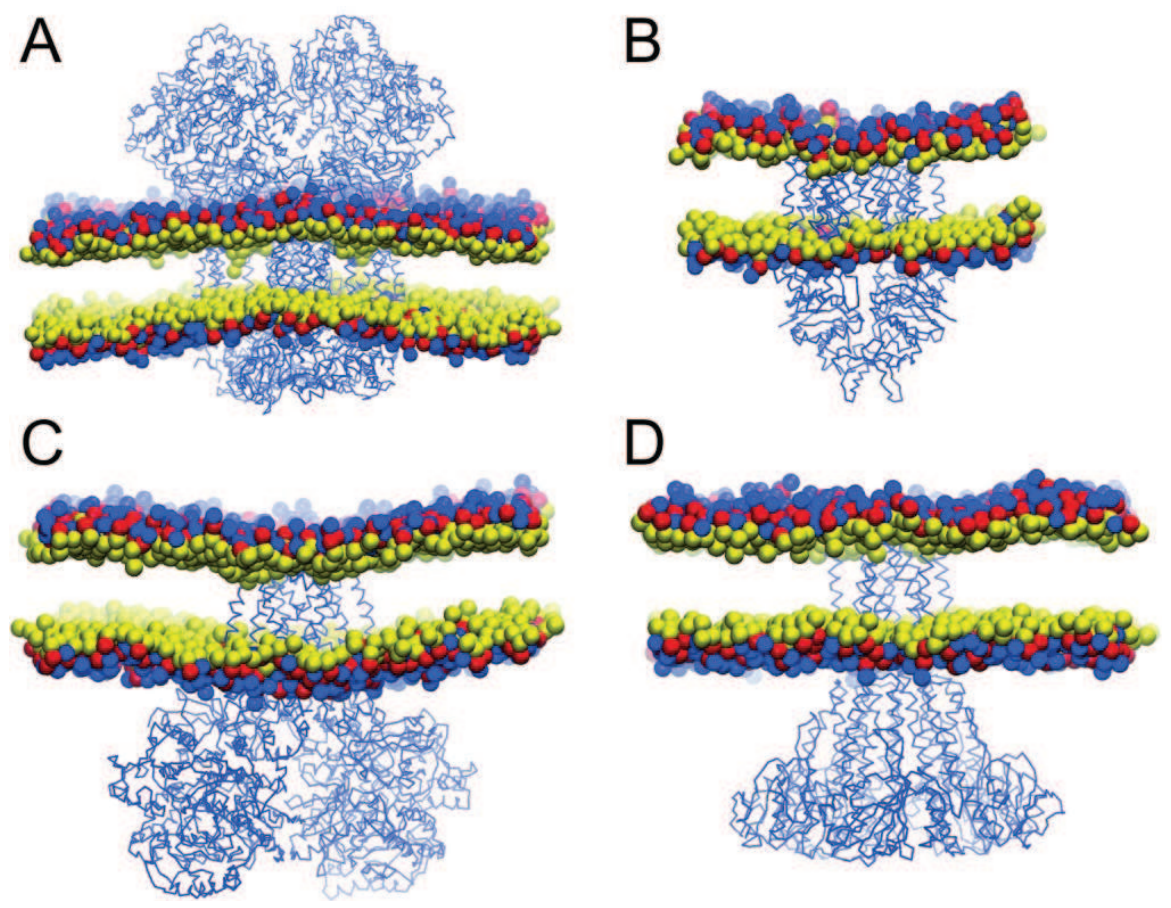

Fig. 5 Resulting snapshots for the final frame in four simulations: A - cytochrome bc1 complex, B - putative metal-chelating $\mathrm{ABC}$ transporter, $\mathrm{C}$ - quinol-fumarate reductase and $\mathrm{D}-\mathrm{Mg}^{2+}$ transporter. Water, ion and DPPC lipid tail particles are excluded for clarity. The backbone trace of the protein is shown in blue. The particle colors are: phosphate in DPPC lipid headgroups: red; glycerol linker in the lipid: yellow; choline in PC headgroups: blue. Picture created based on materials available in the CG Database (Sansom et al. 2008). 
result of the disruption of a lipid bilayer surrounding the protein and less efficient helix-helix packing (Sengupta and Marrink 2010). The observed differences became only apparent after extensive sampling, which indicates the importance of long microsecond simulation timescales.

A multiscale MD approach (combining CG-MD and all-atom-MD simulations) was used by Kalli and coworkers (Kalli et al. 2011) to explore the formation of an aIIb/b3 integrin TM helix hetero-dimer in the DPPC membrane model. CG-MD simulations were performed using high-throughput methodology (Hall et al. 2011) which enabled automatic running of multiple self-assembly simulations and statistical analysis over an ensemble of approximately 100 structures. Dimer formation usually occurred within a few hundred nanoseconds of CG-MD. The resulting dimers were submitted for further assessment and refinement using all-atom-MD simulation. Comparing the final structure of the modeled dimer with the available aIIb/b3 integrin NMR structure (PDB ID: 2K9J (Lau et al. 2009)) yields a C $\alpha$ rmsd of $2.2 \AA$ for the TM region, a similar crossing angle of $30 \pm 3^{\circ}$ and a helixhelix interface created by the same residues. The results indicate that a purely computational based approach may result in hetero-dimer formation with an accuracy similar to the NMR method.

Recently, Periole and coworkers applied large-scale CG-MD simulations to study the energetics of the receptor-receptor dimer interface of the $G$ protein coupled receptor (GPCR), rhodopsin (Periole et al. 2012). The procedure involved self-assembly simulations of multiple copies of rhodopsin embedded into a lipid membrane over time scales ranging from 10 to $100 \mu \mathrm{s}$. During the simulations the potentials of mean force (PMFs) were computed for pairs of rhodopsin molecules along different interfaces. The resulting data pointed to the most stable rhodopsinrhodopsin conformation involving a symmetrical Helix1/Helix8 interface. The observed interface was also in agreement with recent cross-linking experiments (Knepp et al. 2012) and EM density maps (Ruprecht et al. 2004). This approach based on extensive CG-MD simulations may also be used to investigate homoand hetero-dimer interfaces of other members of the GPCR family.

The above examples illustrate some of the CG-MD methods which use the MARTINI force field applied in studies of membrane-protein systems. A wide variety of other CG methods currently exist (Arkhipov et al. 2006; Kolinski 2004) and new force fields are being developed (Zhou et al. 2007; Spijker et al. 2010). CG approaches combined with the Gaussian network model (GNM) and the anisotropy elastic network model (ANM) were also used to investigate the mechanism of L-arginine (Arg)/agmatine (Agm) antiporter (AdiC) (Chang et al. 2010) and prediction of functional motions of outer membrane transporter and signal transducer FecA (Sen et al. 2008). Despite the limitations of CG models, united-atom representation and simplification of energy function, there is a growing need for improving CG computational methods to be used to study the function and dynamics of large and complex protein-membrane systems. Nevertheless, CG-based methods are rapidly advancing and may become invaluable tools for the exploration of some fundamental events that are otherwise still not reachable by biochemical experiments. 


\subsection{Intrinsically Unfolded Proteins}

Over the last decades, the thermodynamically stable conformation of a protein was usually treated as the state responsible for biological functions. Nevertheless, at the end of the $20^{\text {th }}$ century the research community realized that intrinsically disordered proteins (IDP) are ubiquitous in nature and they can retain their functionality (Mittag et al. 2010; Uversky et al. 2000; Wright and Dyson 1999). Conformational studies of these proteins are experimentally extremely challenging (Eliezer 2009), particularly due to their large structural heterogeneity and aggregation tendency. With the boom of IDP studies, computer simulation models have emerged as useful tools for the description of IDP conformational ensembles (Rauscher and Pomès 2010). As the effective search of the conformational space is the major advantage of the CG models, they can be used as methods of choice for possibly the broadest sampling of conformational disorder.

Owing to their flexibility, disordered proteins have increased tendency of forming protein-protein complexes. During binding, as compared to folded structures, IDP's can form a far larger number of interaction contacts and may have a larger capture radius for a specific binding site. This theory is called the "fly-casting mechanism" and it was illustrated by Shoemaker et al. (Shoemaker et al. 2000) who investigated the kinetics of IDP binding to the receptor using their free energy functional based on a simplified scheme of amino acid contacts.

Nevertheless, CG simulations of pKID-KIX complexes (Huang and Liu 2009) indicated that the increased binding affinity can be caused not only by the greater capture radius of IDPs. The kinetic analysis of this process was based on simulations using the CG Go model with the continuum $\mathrm{C}$-alpha chain representation and compared with available experimental data for various ordered and disordered complexes. Interestingly, it was found that the coupling of folding with binding of IDPs leads to a significant reduction in the binding free-energy barrier. This work also discusses roles of other structural factors important for this particular association.

Abeln and Frenkel analyzed other aspects of how intrinsically disordered regions (IDRs) can influence the protein association process using Monte Carlo (MC) simulation on cubic lattice with C-alpha representation (Abeln and Frenkel 2008). The simulation results provided intriguing insights into the effect of IDRs on protein structure. The authors indicated that proteins with hydrophobic binding motifs without neighboring IDRs tend to aggregate and consequently form amyloids.

The ability to fold upon binding of some IDPs has been extensively studied using CG simulation models (Wang et al. 2011; Verkhivker et al. 2003; Verkhivker 2005; Turjanski et al. 2008; De Sancho and Best 2012). A multiscale model was used to generate the pathway of IDP folding induced by binding to its receptor (Wang et al. 2011). The method included a step of CG simulation with C-alpha representation and optimal path calculation at an atomic level. The binding process was simulated as fully flexible and the role of non-native interactions was stressed. In other studies (Verkhivker et al. 2003; Verkhivker 2005) the authors characterized an ensemble of transition states of p27Kip1 protein binding to a rigid structure of a cyclin A - Cdk2 complex. In this case a knowledge-based 
potential was utilized to investigate some aspects of the folding mechanism of this protein. Intrinsically disordered proteins frequently serve as flexible linkers of protein domains. CG modeling of such systems was reviewed by Zhou (Zhou 2004).

Similar to protein structure prediction, IDP modeling approaches can be divided into de novo methods (based on the prediction power of the method) and those utilizing sparse experimental data. The CG C-alpha model of Norgaard et al. (Norgaard et al. 2008) was designed to simulate disordered proteins and parameterized using data from nuclear magnetic resonance spin-labeling experiments on the $\Delta 131 \Delta$ fragment of Staphylococcal nuclease. Importantly, such an approach can be used by utilizing data from MD trajectories or other experiments.

Interestingly, 2D lattice models have been recently used to explain the worse performance of sequence-based disorder prediction methods for smaller proteins (or segments) than for larger ones. Such a simple simulation model enabled a novel insight into the basic determinants of protein disorder: amino acid composition and chain length (Szilagyi et al. 2008).

As shown above, CG models, even very simplistic ones, provided many important facts for the description of IDP and IDR dynamics. However, the potential of CG modeling does not seem to be sufficiently exploited in the field (Rauscher and Pomes 2010), perhaps because of the relatively recent interest in the area.

\section{Conclusions and Perspectives}

An obvious advantage of $\mathrm{CG}$ protein simulations is that larger protein systems can be studied and longer timescales can be assessed than possible with atomicresolution MD. Apart from expanding the limits, the speed-up benefit of CG models brings many new opportunities for the design of some extensive 'in silico experiments' (Takada 2012), such as: comparative dynamics for a large set of proteins (Takagi et al. 2003), comprehensive mutation analysis (Koga and Takada 2006), scanning parameters of a simulation model to see how it affects simulation results (Yao et al. 2010) or construction of databases by high-throughput simulation protocols (Chetwynd et al. 2008).

CG protein modeling has already a history of a few decades (Kolinski and Skolnick 2004). The last decade showed a dramatic increase in CG modeling studies of large biomolecules (Takada 2012). We can expect that this trend will continue in the foreseeable future, since atomic-resolution MD is far too slow for current needs. The current need for computer-enhanced studies of large biomolecules is largely due to the recent growth of experimental data on their structure and, consequently, the need for data interpretation and validation of resulting hypotheses.

In this chapter, we described recent applications of $\mathrm{CG}$ simulations to some representative and important topics of protein dynamics. The work demonstrates the utility of CG modeling in understanding real biological problems. As shown, there are many variants of CG simulation tools and many successful strategies in which CG models are an important component. Future developments are expected 
to include CG models in unified/integrative structure modeling procedures utilizing a wide range of experimental and computational techniques (Russel et al. 2009; Schlick et al. 2011). Consequently, the integration of protein CG models together with CG models for other molecules (lipids, nucleic acids, carbohydrates) as well as CG models with atomic MD (so-called multiscale approach) should be the focus of further research.

Acknowledgments. We thank dr. Joanna Sulkowska for critical reading of the section "Mechanical unfolding of proteins" of the manuscript. We acknowledge partial support from: Foundation for Polish Science TEAM project (TEAM/2011-7/6) co-financed by the European Regional Development Fund operated within the Innovative Economy Operational Program; Polish National Science Center $(\mathrm{NCN})$ on the basis of a decision DEC2011/01/D/NZ2/05314; Polish National Science Center (NCN) Grant No. NN301071140, Polish Ministry of Science and Higher Education Grant No. IP2011024371.

\section{References}

Abeln, S., Frenkel, D.: Disordered Flanks Prevent Peptide Aggregation. PLoS Comput. Biol. 4(12), e1000241 (2008), doi:10.1371/journal.pcbi.1000241

Arad-Haase, G., Chuartzman, S.G., Dagan, S., Nevo, R., Kouza, M., Mai, B.K., Nguyen, H.T., Li, M.S., Reich, Z.: Mechanical unfolding of acylphosphatase studied by singlemolecule force spectroscopy and MD simulations. Biophys. J. 99(1), 238-247 (2010), doi:10.1016/j.bpj.2010.04.004

Arkhipov, A., Freddolino, P.L., Schulten, K.: Stability and dynamics of virus capsids described by coarse-grained modeling. Structure 14(12), 1767-1777 (2006), doi:10.1016/j.str.2006.10.003

Auer, S., Meersman, F., Dobson, C.M., Vendruscolo, M.: A Generic Mechanism of Emergence of Amyloid Protofilaments from Disordered Oligomeric Aggregates. PLoS Comput. Biol. 4(11), e1000222 (2008), doi:10.1371/journal.pcbi.1000222

Baumketner, A., Jewett, A., Shea, J.E.: Effects of confinement in chaperonin assisted protein folding: rate enhancement by decreasing the roughness of the folding energy landscape. J. Mol. Biol. 332(3), 701-713 (2003), doi:10.1016/S0022-2836(03)00929-X

Bell, G.I.: Models for the specific adhesion of cells to cells. Science 200(4342), 618-627 (1978), doi:10.1126/science.347575

Best, R.B., Hummer, G.: Protein folding kinetics under force from molecular simulation. J. Am. Chem. Soc. 130(12), 3706-3707 (2008), doi:10.1021/ja0762691

Best, R.B., Paci, E., Hummer, G., Dudko, O.K.: Pulling direction as a reaction coordinate for the mechanical unfolding of single molecules. Journal of Physical Chemistry B 112(19), 5968-5976 (2008), doi:10.1021/Jp075955j

Betancourt, M.R., Thirumalai, D.: Exploring the kinetic requirements for enhancement of protein folding rates in the GroEL cavity. J. Mol. Biol. 287(3), 627-644 (1999), doi:10.1006/jmbi.1999.2591

Bindschadler, M.: Modeling actin dynamics. Wiley Interdisciplinary Reviews: Systems Biology and Medicine 2(4), 481-488 (2010), doi:10.1002/wsbm.62

Brockwell, D.J., Paci, E., Zinober, R.C., Beddard, G.S., Olmsted, P.D., Smith, D.A., Perham, R.N., Radford, S.E.: Pulling geometry defines the mechanical resistance of a betasheet protein (vol 10, pg 731, 2003). Nature Structural Biology 10(10), 872-872 (2003), doi:10.1038/Nsb1003-872b 
Bustamante, C., Chemla, Y.R., Forde, N.R., Izhaky, D.: Mechanical processes in biochemistry. Annu. Rev. Biochem. 73, 705-748 (2004), doi:10.1146/annurev.biochem.72.121801.161542

Caraglio, M., Imparato, A., Pelizzola, A.: Pathways of mechanical unfolding of FnIII(10): low force intermediates. J. Chem. Phys. 133(6), 065101 (2010), doi:10.1063/1.3464476

Carrion-Vazquez, M., Li, H., Lu, H., Marszalek, P.E., Oberhauser, A.F., Fernandez, J.M.: The mechanical stability of ubiquitin is linkage dependent. Nat. Struct. Biol. 10(9), 738743 (2003), doi:10.1038/nsb965

Chang, S., Hu, J.P., Lin, P.Y., Jiao, X., Tian, X.H.: Substrate recognition and transport behavior analyses of amino acid antiporter with coarse-grained models. Mol. Biosyst. 6(12), 2430-2438 (2010), doi:10.1039/c005266c

Chetwynd, A.P., Scott, K.A., Mokrab, Y., Sansom, M.S.: CGDB: a database of membrane protein/lipid interactions by coarse-grained molecular dynamics simulations. Mol. Membr. Biol. 25(8), 662-669 (2008), doi:10.1080/09687680802446534

Chu, J.W., Voth, G.A.: Coarse-grained modeling of the actin filament derived from atomistic-scale simulations. Biophys. J. 90(5), 1572-1582 (2006), doi:10.1529/biophysj.105.073924

Cieplak, M., Hoang, T.X., Robbins, M.O.: Folding and stretching in a Go-like model of titin. Proteins 49(1), 114-124 (2002), doi:10.1002/prot.10087

Clementi, C., Nymeyer, H., Onuchic, J.N.: Topological and energetic factors: what determines the structural details of the transition state ensemble and "en-route" intermediates for protein folding? An investigation for small globular proteins. J. Mol. Biol. 298(5), 937-953 (2000), doi:10.1006/jmbi.2000.3693

De Sancho, D., Best, R.B.: Modulation of an IDP binding mechanism and rates by helix propensity and non-native interactions: association of HIF1alpha with CBP. Mol Biosyst. 8(1), 256-267 (2012), doi:10.1039/c1 mb05252g

Di Fenza, A., Rocchia, W., Tozzini, V.: Complexes of HIV-1 integrase with HAT proteins: Multiscale models, dynamics, and hypotheses on allosteric sites of inhibition. Proteins: Structure, Function, and Bioinformatics 76(4), 946-958 (2009), doi:10.1002/prot.22399

Dudko, O.K., Hummer, G., Szabo, A.: Intrinsic rates and activation free energies from single-molecule pulling experiments. Phys. Rev. Lett. 96(10), 108101 (2006), doi:10.1103/PhysRevLett.96.108101

Eliezer, D.: Biophysical characterization of intrinsically disordered proteins. Current Opinion in Structural Biology 19(1), 23-30 (2009), doi:10.1016/j.sbi.2008.12.004

Evans, E., Ritchie, K.: Dynamic strength of molecular adhesion bonds. Biophys. J. 72(4), 1541-1555 (1997), doi:10.1016/S0006-3495(97)78802-7

Fletcher, D.A., Mullins, R.D.: Cell mechanics and the cytoskeleton. Nature 463(7280), 485-492 (2010), doi:10.1038/nature08908

Florin, E.L., Moy, V.T., Gaub, H.E.: Adhesion forces between individual ligand-receptor pairs. Science 264(5157), 415-417 (1994), doi:10.1126/science.8153628

Fowler, S.B., Best, R.B., Toca Herrera, J.L., Rutherford, T.J., Steward, A., Paci, E., Karplus, M., Clarke, J.: Mechanical unfolding of a titin Ig domain: structure of unfolding intermediate revealed by combining AFM, molecular dynamics simulations, NMR and protein engineering. J. Mol. Biol. 322(4), 841-849 (2002), doi:10.1016/S00222836(02)00805-7

Frembgen-Kesner, T., Elcock, A.H.: Absolute Protein-Protein Association Rate Constants from Flexible, Coarse-Grained Brownian Dynamics Simulations: The Role of Intermolecular Hydrodynamic Interactions in Barnase-Barstar Association. Biophys. J. 99(9), L75-L77 (2010), doi:10.1016/j.bpj.2010.09.006 
Granzier, H.L., Labeit, S.: The giant protein titin: a major player in myocardial mechanics, signaling, and disease. Circ. Res. 94(3), 284-295 (2004), doi:10.1161/01.RES.0000117769.88862.F8

Hall, B.A., Chetwynd, A.P., Sansom, M.S.: Exploring peptide-membrane interactions with coarse-grained MD simulations. Biophys. J. 100(8), 1940-1948 (2011), doi:10.1016/j.bpj.2011.02.041

Hall, B.A., Sansom, M.S.P.: Coarse-Grained MD Simulations and Protein-Protein Interactions: The Cohesin-Dockerin System. Journal of Chemical Theory and Computation 5(9), 2465-2471 (2009), doi:10.1021/ct900140w

Hanson, P.I., Whiteheart, S.W.: AAA+ proteins: have engine, will work. Nat. Rev. Mol. Cell Biol. 6(7), 519-529 (2005), doi:10.1038/nrm1684

He, C., Genchev, G.Z., Lu, H., Li, H.: Mechanically untying a protein slipknot: multiple pathways revealed by force spectroscopy and steered molecular dynamics simulations. J. Am. Chem. Soc. 134(25), 10428-10435 (2012), doi:10.1021/ja3003205

Heath, A.P., Kavraki, L.E., Clementi, C.: From coarse-grain to all-atom: toward multiscale analysis of protein landscapes. Proteins 68(3), 646-661 (2007), doi:10.1002/prot.21371

Huang, Y., Liu, Z.: Kinetic Advantage of Intrinsically Disordered Proteins in Coupled Folding-Binding Process: A Critical Assessment of the "Fly-Casting" Mechanism. Journal of Molecular Biology 393(5), 1143-1159 (2009), doi:10.1016/j.jmb.2009.09.010

Hunte, C.: Specific protein-lipid interactions in membrane proteins. Biochem. Soc. Trans. 33(Pt. 5), 938-942 (2005), doi:10.1042/BST20050938

Irback, A., Mitternacht, S., Mohanty, S.: Dissecting the mechanical unfolding of ubiquitin. Proc. Natl. Acad. Sci. U S A 102(38), 13427-13432 (2005), doi:10.1073/pnas.0501581102

Jacob, E., Horovitz, A., Unger, R.: Different mechanistic requirements for prokaryotic and eukaryotic chaperonins: a lattice study. Bioinformatics 23(13), i240-i248 (2007), doi:10.1093/bioinformatics/btm180

Jewett, A.I., Baumketner, A., Shea, J.E.: Accelerated folding in the weak hydrophobic environment of a chaperonin cavity: creation of an alternate fast folding pathway. Proc. Natl. Acad. Sci. U S A 101(36), 13192-13197 (2004), doi:10.1073/pnas.0400720101

Jewett, A.I., Shea, J.E.: Reconciling theories of chaperonin accelerated folding with experimental evidence. Cell. Mol. Life Sci. 67(2), 255-276 (2009)

Kalli, A.C., Hall, B.A., Campbell, I.D., Sansom, M.S.: A helix heterodimer in a lipid bilayer: prediction of the structure of an integrin transmembrane domain via multiscale simulations. Structure 19(10), 1477-1484 (2011), doi:10.1016/j.str.2011.07.014

Kamerlin, S.C., Vicatos, S., Dryga, A., Warshel, A.: Coarse-grained (multiscale) simulations in studies of biophysical and chemical systems. Annual Review of Physical Chemistry 62, 41-64 (2011), doi:10.1146/annurev-physchem-032210-103335

Kim, Y.C., Hummer, G.: Coarse-grained Models for Simulations of Multiprotein Complexes: Application to Ubiquitin Binding. Journal of Molecular Biology 375(5), 1416-1433 (2008), doi:10.1016/j.jmb.2007.11.063

Kim, Y.C., Tang, C., Clore, G.M., Hummer, G.: Replica exchange simulations of transient encounter complexes in protein-protein association. Proc. Natl. Acad. Sci. U S A 105(35), 12855-12860 (2008), doi:10.1073/pnas.0802460105

Kmiecik, S., Gront, D., Kolinski, A.: Towards the high-resolution protein structure prediction. Fast refinement of reduced models with all-atom force field. Bmc Struct. Biol. 7(43), 43 (2007), doi:10.1186/1472-6807-7-43 
Kmiecik, S., Gront, D., Kouza, M., Kolinski, A.: From coarse-grained to atomic-level characterization of protein dynamics: transition state for the folding of $\mathrm{B}$ domain of protein A. J. Phys. Chem. B 116(23), 7026-7032 (2012), doi:10.1021/jp301720w

Kmiecik, S., Jamroz, M., Kolinski, A.: Multiscale Approach to Protein Folding Dynamics. In: Kolinski, A. (ed.) Multiscale Approaches to Protein Modeling, pp. 281-293. Springer, New York (2011), doi:10.1007/978-1-4419-6889-0_12

Kmiecik, S., Kolinski, A.: Folding pathway of the b1 domain of protein G explored by multiscale modeling. Biophys. J. 94(3), 726-736 (2008), doi:10.1529/biophysj.107.116095

Kmiecik, S., Kolinski, A.: Simulation of chaperonin effect on protein folding: a shift from nucleation-condensation to framework mechanism. J. Am. Chem. Soc. 133(26), 1028310289 (2011), doi:10.1021/ja203275f

Knepp, A.M., Periole, X., Marrink, S.J., Sakmar, T.P., Huber, T.: Rhodopsin forms a dimer with cytoplasmic helix 8 contacts in native membranes. Biochemistry 51(9), 1819-1821 (2012), doi:10.1021/bi3001598

Koga, N., Takada, S.: Folding-based molecular simulations reveal mechanisms of the rotary motor F1-ATPase. Proc. Natl. Acad. Sci. U S A 103(14), 5367-5372 (2006), doi:10.1073/pnas.0509642103

Kolinski, A.: Protein modeling and structure prediction with a reduced representation. Acta Biochimica Polonica 51(2), 349-371 (2004), doi:035001349

Kolinski, A., Skolnick, J.: Reduced models of proteins and their applications. Polymer 45(2), 511-524 (2004), doi:10.1016/j.polymer.2003.10.064

Kouza, M., Hu, C.K., Li, M.S.: New force replica exchange method and protein folding pathways probed by force-clamp technique. J. Chem. Phys. 128(4), 045103 (2008), doi:10.1063/1.2822272

Kouza, M., Hu, C.K., Zung, H., Li, M.S.: Protein mechanical unfolding: Importance of non-native interactions. J. Chem. Phys. 131(21), 215103 (2009), doi:10.1063/1.3272275

Kramers, H.A.: Brownian motion in a field of force and the diffusion model of chemical reactions. Physica 7(7), 284-303 (1940), doi:10.1016/S0031-8914(40)90098-2

Kumar, S., Li, M.S.: Biomolecules under mechanical force. Phys. Rep. 486(1-2), 1-74 (2010), doi:10.1016/j.physrep.2009.11.001

Kurcinski, M., Kolinski, A.: Theoretical study of molecular mechanism of binding TRAP220 coactivator to Retinoid X Receptor alpha, activated by 9-cis retinoic acid. The Journal of Steroid Biochemistry and Molecular Biology 121(1-2), 124-129 (2010), doi:10.1016/j.jsbmb.2010.03.086

Lau, T.L., Kim, C., Ginsberg, M.H., Ulmer, T.S.: The structure of the integrin alphallbbeta3 transmembrane complex explains integrin transmembrane signalling. Embo. J. 28(9), 1351-1361 (2009), doi:10.1038/emboj.2009.63

Lee, A.G.: How lipids affect the activities of integral membrane proteins. BbaBiomembranes 1666(1-2), 62-87 (2004), doi:10.1016/j.bbamem.2004.05.012

Lee, E.H., Hsin, J., Sotomayor, M., Comellas, G., Schulten, K.: Discovery Through the Computational Microscope. Structure 17(10), 1295-1306 (2009), doi:10.1016/j.str.2009.09.001

Levitt, M., Warshel, A.: Computer simulation of protein folding. Nature 253(5494), 694698 (1975), doi:10.1038/253694a0

Li, L., Huang, H.H., Badilla, C.L., Fernandez, J.M.: Mechanical unfolding intermediates observed by single-molecule force spectroscopy in a fibronectin type III module. J. Mol. Biol. 345(4), 817-826 (2005), doi:10.1016/j.jmb.2004.11.021 
Li, M.S.: Secondary structure, mechanical stability, and location of transition state of proteins. Biophys. J. 93(8), 2644-2654 (2007), doi:10.1529/biophysj.107.106138

Li, M.S., Kouza, M.: Dependence of protein mechanical unfolding pathways on pulling speeds. J. Chem. Phys. 130(14), 145102 (2009), doi:10.1063/1.3106761

Li, M.S., Kouza, M., Hu, C.K.: Refolding upon force quench and pathways of mechanical and thermal unfolding of ubiquitin. Biophys. J. 92(2), 547-561 (2007), doi:10.1529/biophysj.106.087684

Lichter, S., Rafferty, B., Flohr, Z., Martini, A.: Protein high-force pulling simulations yield low-force results. PLoS One 7(4), e34781 (2012), doi:10.1371/journal.pone.0034781

Liphardt, J., Onoa, B., Smith, S.B., Tinoco Jr., I., Bustamante, C.: Reversible unfolding of single RNA molecules by mechanical force. Science 292(5517), 733-737 (2001), doi:10.1126/science. 1058498

Lu, H., Isralewitz, B., Krammer, A., Vogel, V., Schulten, K.: Unfolding of titin immunoglobulin domains by steered molecular dynamics simulation. Biophys. J. 75(2), 662-671 (1998), doi:10.1016/S0006-3495(98)77556-3

Lu, H., Schulten, K.: The key event in force-induced unfolding of Titin's immunoglobulin domains. Biophys. J. 79(1), 51-65 (2000), doi:10.1016/S0006-3495(00)76273-4

Lucent, D., England, J., Pande, V.: Inside the chaperonin toolbox: theoretical and computational models for chaperonin mechanism. Physical Biology 6(1), 015003 (2009), doi:10.1088/1478-3975/6/1/015003

Marrink, S.J., Risselada, H.J., Yefimov, S., Tieleman, D.P., de Vries, A.H.: The MARTINI force field: coarse grained model for biomolecular simulations. J. Phys. Chem. B 111(27), 7812-7824 (2007), doi:10.1021/jp071097f

Marszalek, P.E., Lu, H., Li, H., Carrion-Vazquez, M., Oberhauser, A.F., Schulten, K., Fernandez, J.M.: Mechanical unfolding intermediates in titin modules. Nature 402(6757), 100-103 (1999), doi:10.1038/47083

Mittag, T., Kay, L.E., Forman-Kay, J.D.: Protein dynamics and conformational disorder in molecular recognition. Journal of Molecular Recognition 23(2), 105-116 (2010), doi:10.1002/jmr.961

Munoz, V., Henry, E.R., Hofrichter, J., Eaton, W.A.: A statistical mechanical model for beta-hairpin kinetics. Proc. Natl. Acad. Sci. U S A 95(11), 5872-5879 (1998), doi:10.1073/pnas.95.11.5872

Nilsson, J., Persson, B., von Heijne, G.: Comparative analysis of amino acid distributions in integral membrane proteins from 107 genomes. Proteins 60(4), 606-616 (2005), doi:10.1002/prot.20583

Norgaard, A.B., Ferkinghoff-Borg, J., Lindorff-Larsen, K.: Experimental Parameterization of an Energy Function for the Simulation of Unfolded Proteins. Biophys. J. 94(1), 182192 (2008), doi:10.1529/biophysj.107.108241

Okazaki, K.-I., Sato, T., Takano, M.: Temperature-Enhanced Association of Proteins Due to Electrostatic Interaction: A Coarse-Grained Simulation of Actin-Myosin Binding. J. Am. Chem. Soc. 134(21), 8918-8925 (2012), doi:10.1021/ja301447j

Paci, E., Karplus, M.: Unfolding proteins by external forces and temperature: the importance of topology and energetics. Proc. Natl. Acad. Sci. U S A 97(12), 6521-6526 (2000), doi:10.1073/pnas.100124597

Peplowski, L., Sikora, M., Nowak, W., Cieplak, M.: Molecular jamming-the cystine slipknot mechanical clamp in all-atom simulations. J. Chem. Phys. 134(8), 085102 (2011), doi:10.1063/1.3553801 
Periole, X., Knepp, A.M., Sakmar, T.P., Marrink, S.J., Huber, T.: Structural determinants of the supramolecular organization of $G$ protein-coupled receptors in bilayers. J. Am. Chem. Soc. 134(26), 10959-10965 (2012), doi:10.1021/ja303286e

Plaxco, K.W., Simons, K.T., Baker, D.: Contact order, transition state placement and the refolding rates of single domain proteins. J. Mol. Biol. 277(4), 985-994 (1998), doi:10.1006/jmbi.1998.1645

Rathore, N., Knotts IV, T.A., de Pablo, J.J.: Confinement effects on the thermodynamics of protein folding: Monte Carlo simulations. Biophys J. 90(5), 1767-1773 (2006), doi:10.1529/biophysj.105.071076

Rauscher, S., Pomes, R.: Molecular simulations of protein disorder. Biochem. Cell. Biol. 88(2), 269-290 (2010), doi:10.1139/o09-169

Rauscher, S., Pomès, R.: Molecular simulations of protein disorderThis paper is one of a selection of papers published in this special issue entitled "Canadian Society of Biochemistry, Molecular \& Cellular Biology 52nd Annual Meeting — Protein Folding: Principles and Diseases" and has undergone the Journal's usual peer review process. Biochemistry and Cell Biology 88(2), 269-290 (2010), doi:10.1139/o09-169

Rief, M., Gautel, M., Oesterhelt, F., Fernandez, J.M., Gaub, H.E.: Reversible unfolding of individual titin immunoglobulin domains by AFM. Science 276(5315), 1109-1112 (1997), doi:10.1126/science.276.5315.1109

Ruprecht, J.J., Mielke, T., Vogel, R., Villa, C., Schertler, G.F.: Electron crystallography reveals the structure of metarhodopsin I. Embo J. 23(18), 3609-3620 (2004), doi:10.1038/sj.emboj.7600374

Russel, D., Lasker, K., Phillips, J., Schneidman-Duhovny, D., Velazquez-Muriel, J.A., Sali, A.: The structural dynamics of macromolecular processes. Current Opinion in Cell Biology 21(1), 97-108 (2009), doi:10.1016/j.ceb.2009.01.022

Sansom, M.S., Scott, K.A., Bond, P.J.: Coarse-grained simulation: a high-throughput computational approach to membrane proteins. Biochem. Soc. Trans. 36(Pt. 1), 27-32 (2008), doi:10.1042/BST0360027

Saunders, M.G., Voth, G.A.: Coarse-graining of multiprotein assemblies. Current Opinion in Structural Biology 22(2), 144-150 (2012), doi:10.1016/j.sbi.2012.01.003

Scheraga, H.A., Khalili, M., Liwo, A.: Protein-folding dynamics: overview of molecular simulation techniques. Annual Review of Physical Chemistry 58, 57-83 (2007), doi:10.1146/annurev.physchem.58.032806.104614

Schlick, T., Collepardo-Guevara, R., Halvorsen, L.A., Jung, S., Xiao, X.: Biomolecularmodeling and simulation: a field coming of age. Q. Rev. Biophys. 44(2), 191-228 (2011), doi:10.1017/S0033583510000284

Schwaiger, I., Kardinal, A., Schleicher, M., Noegel, A.A., Rief, M.: A mechanical unfolding intermediate in an actin-crosslinking protein. Nat. Struct. Mol. Biol. 11(1), 81-85 (2004), doi:10.1038/nsmb705

Scott, K.A., Bond, P.J., Ivetac, A., Chetwynd, A.P., Khalid, S., Sansom, M.S.: Coarsegrained MD simulations of membrane protein-bilayer self-assembly. Structure 16(4), 621-630 (2008), doi:10.1016/j.str.2008.01.014

Sen, T.Z., Kloster, M., Jernigan, R.L., Kolinski, A., Bujnicki, J.M., Kloczkowski, A.: Predicting the complex structure and functional motions of the outer membrane transporter and signal transducer FecA. Biophys. J. 94(7), 2482-2491 (2008), doi:10.1529/biophysj.107.116046 
Sengupta, D., Marrink, S.J.: Lipid-mediated interactions tune the association of glycophorin A helix and its disruptive mutants in membranes. Phys. Chem. Chem. Phys. 12(40), 12987-12996 (2010), doi:10.1039/c0cp00101e

Serohijos, A.W., Chen, Y., Ding, F., Elston, T.C., Dokholyan, N.V.: A structural model reveals energy transduction in dynein. Proc. Natl. Acad. Sci. U S A 103(49), 1854018545 (2006), doi:10.1073/pnas.0602867103

Shoemaker, B.A., Portman, J.J., Wolynes, P.G.: Speeding molecular recognition by using the folding funnel: The fly-casting mechanism. Proceedings of the National Academy of Sciences 97(16), 8868-8873 (2000), doi:10.1073/pnas.160259697

Sieben, C., Kappel, C., Zhu, R., Wozniak, A., Rankl, C., Hinterdorfer, P., Grubmüller, H., Herrmann, A.: Influenza virus binds its host cell using multiple dynamic interactions. Proc. Natl. Acad. Sci. U S A 109(34), 13626-13631 (2012), doi:10.1073/pnas.1120265109

Sikora, M., Cieplak, M.: Mechanical stability of multidomain proteins and novel mechanical clamps. Proteins 79(6), 1786-1799 (2011), doi:10.1002/prot.23001

Sikora, M., Sulkowska, J.I., Witkowski, B.S., Cieplak, M.: BSDB: the biomolecule stretching database. Nucleic Acids Res. 39(Database Issue), D443-D450 (2011), doi:10.1093/nar/gkq851

Simmons, R.M., Finer, J.T., Chu, S., Spudich, J.A.: Quantitative measurements of force and displacement using an optical trap. Biophys. J. 70(4), 1813-1822 (1996), doi:10.1016/S0006-3495(96)79746-1

Smith, S.B., Cui, Y., Bustamante, C.: Overstretching B-DNA: the elastic response of individual double-stranded and single-stranded DNA molecules. Science 271(5250), 795799 (1996), doi:10.1126/science.271.5250.795

Smith, S.O., Eilers, M., Song, D., Crocker, E., Ying, W., Groesbeek, M., Metz, G., Ziliox, M., Aimoto, S.: Implications of threonine hydrogen bonding in the glycophorin A transmembrane helix dimer. Biophys. J. 82(5), 2476-2486 (2002), doi:10.1016/S0006-3495(02)75590-2

Spijker, P., van Hoof, B., Debertrand, M., Markvoort, A.J., Vaidehi, N., Hilbers, P.A.: Coarse grained molecular dynamics simulations of transmembrane protein-lipid systems. Int. J. Mol. Sci. 11(6), 2393-2420 (2010), doi:10.3390/ijms11062393

Stossel, T.P., Condeelis, J., Cooley, L., Hartwig, J.H., Noegel, A., Schleicher, M., Shapiro, S.S.: Filamins as integrators of cell mechanics and signalling. Nat. Rev. Mol. Cell. Biol. 2(2), 138-145 (2001), doi:10.1038/35052082

Sulkowska, J.I., Cieplak, M.: Mechanical stretching of proteins - a theoretical survey of the Protein Data Bank. J. Phys.-Condens Mat. 19(28) (2007), doi:10.1088/0953-8984/19/28/283201

Sulkowska, J.I., Sulkowski, P., Onuchic, J.N.: Jamming proteins with slipknots and their free energy landscape. Phys. Rev. Lett. 103(26), 268103 (2009), doi:10.1103/PhysRevLett.103.268103

Sulkowska, J.I., Sulkowski, P., Szymczak, P., Cieplak, M.: Untying knots in proteins. J. Am. Chem. Soc. 132(40), 13954-13956 (2010), doi:10.1021/ja102441z

Szilagyi, A., Gyorffy, D., Zavodszky, P.: The twilight zone between protein order and disorder. Biophys. J. 95(4), 1612-1626 (2008), doi:10.1529/biophysj.108.131151

Szymczak, P., Janovjak, H.: Periodic forces trigger a complex mechanical response in ubiquitin. J. Mol. Biol. 390(3), 443-456 (2009), doi:10.1016/j.jmb.2009.04.071 
Takada, S.: Coarse-grained molecular simulations of large biomolecules. Curr. Opin. Struct. Biol. 22(2), 130-137 (2012), doi:10.1016/j.sbi.2012.01.010

Takagi, F., Koga, N., Takada, S.: How protein thermodynamics and folding mechanisms are altered by the chaperonin cage: Molecular simulations. Proc. Natl. Acad. Sci. USA 100(20), 11367-11372 (2003), doi:10.1073/pnas.1831920100

Taylor, W.R., Katsimitsoulia, Z.: A coarse-grained molecular model for actin-myosin simulation. J. Mol. Graph. Model. 29(2), 266-279 (2010), doi:10.1016/j.jmgm.2010.06.004

Turjanski, A.G., Gutkind, J.S., Best, R.B., Hummer, G.: Binding-Induced Folding of a Natively Unstructured Transcription Factor. PLoS Comput. Biol. 4(4), e1000060 (2008), doi:10.1371/journal.pcbi.1000060

Uversky, V.N., Gillespie, J.R., Fink, A.L.: Why are "natively unfolded" proteins unstructured under physiologic conditions? Proteins: Structure, Function, and Bioinformatics 41(3), 415-427 (2000), doi:10.1002/1097-0134(20001115)41:3<415::aid-prot130>3.0.co;2-7

Vajda, S., Kozakov, D.: Convergence and combination of methods in protein-protein docking. Curr. Opin. Struct. Biol. 19(2), 164-170 (2009), doi:10.1016/j.sbi.2009.02.008

Valbuena, A., Oroz, J., Hervas, R., Vera, A.M., Rodriguez, D., Menendez, M., Sulkowska, J.I., Cieplak, M., Carrion-Vazquez, M.: On the remarkable mechanostability of scaffoldins and the mechanical clamp motif. Proc. Natl. Acad. Sci. U S A 106(33), 1379113796 (2009), doi:10.1073/pnas.0813093106

Vendruscolo, M., Dobson, C.M.: Protein Dynamics: Moore's Law in Molecular Biology. Curr. Biol. 21(2), R68-R70 (2011), doi:10.1016/j.cub.2010.11.062

Verkhivker, G.M.: Protein conformational transitions coupled to binding in molecular recognition of unstructured proteins: Deciphering the effect of intermolecular interactions on computational structure prediction of the $\mathrm{p} 27 \mathrm{Kip} 1$ protein bound to the cyclin Acyclin-dependent kinase 2 complex. Proteins: Structure, Function, and Bioinformatics 58(3), 706-716 (2005), doi:10.1002/prot.20351

Verkhivker, G.M., Bouzida, D., Gehlhaar, D.K., Rejto, P.A., Freer, S.T., Rose, P.W.: Simulating disorder-order transitions in molecular recognition of unstructured proteins: Where folding meets binding. Proceedings of the National Academy of Sciences 100(9), 5148-5153 (2003), doi:10.1073/pnas.0531373100

Vogel, V., Sheetz, M.: Local force and geometry sensing regulate cell functions. Nat. Rev. Mol. Cell. Biol. 7(4), 265-275 (2006), doi:10.1038/nrm1890

Wang, J., Wang, Y., Chu, X., Hagen, S.J., Han, W., Wang, E.: Multi-Scaled Explorations of Binding-Induced Folding of Intrinsically Disordered Protein Inhibitor IA3 to its Target Enzyme. PLoS Comput. Biol. 7(4), e1001118 (2011), doi:10.1371/journal.pcbi.1001118

West, D.K., Olmsted, P.D., Paci, E.: Mechanical unfolding revisited through a simple but realistic model. J. Chem. Phys. 124(15) (2006), doi:10.1063/1.2185100

Wolynes, P.G., Onuchic, J.N., Thirumalai, D.: Navigating the folding routes. Science 267(5204), 1619-1620 (1995), doi:10.1126/science.7886447

Wright, P.E., Dyson, H.J.: Intrinsically unstructured proteins: re-assessing the protein structure-function paradigm. J. Mol. Biol. 293(2), 321-331 (1999), doi:10.1006/jmbi.1999.3110

Wu, C., Shea, J.E.: Coarse-grained models for protein aggregation. Curr. Opin. Struct. Biol. 21(2), 209-220 (2011), doi:10.1016/j.sbi.2011.02.002 
Yao, X.Q., Kenzaki, H., Murakami, S., Takada, S.: Drug export and allosteric coupling in a multidrug transporter revealed by molecular simulations. Nat. Commun. 1, 117 (2010), doi:10.1038/ncomms1116

Zhang, J., Muthukumar, M.: Simulations of nucleation and elongation of amyloid fibrils. J. Chem. Phys. 130(3), 035102 (2009), doi:10.1063/1.3050295

Zhmurov, A., Dima, R.I., Kholodov, Y., Barsegov, V.: Sop-GPU: accelerating biomolecular simulations in the centisecond timescale using graphics processors. Proteins 78(14), 2984-2999 (2010), doi:10.1002/prot.22824

Zhou, H.-X.: Polymer Models of Protein Stability, Folding, and Interactions $\dagger$. Biochemistry 43(8), 2141-2154 (2004), doi:10.1021/bi036269n

Zhou, H.X., Dill, K.A.: Stabilization of proteins in confined spaces. Biochemistry 40(38), 11289-11293 (2001), doi:10.3410/f.1002736.29765

Zhou, J., Thorpe, I.F., Izvekov, S., Voth, G.A.: Coarse-grained peptide modeling using a systematic multiscale approach. Biophys. J. 92(12), 4289-4303 (2007), doi:10.1529/biophysj.106.094425 